\title{
"Hospital at home" care was as effective as routine hospital care for older adults
}

\author{
Richards SH, Coast J, Gunnell DJ, et al. Randomised controlled trial comparing effectiveness and acceptability of an early discharge, \\ hospital at home scheme with acute hospital care. BMJ 1998 Jun 13;316:1796-801.
}

\section{Coast J, Richards SH, Peters TJ, et al. Hospital at home or acute hospital care? A cost minimisation analysis. BMJ 1998 Jun 13;316:1802-6.}

\section{Question}

Is "hospital at home" $(\mathrm{HaH})$ care as effective and cost effective as inpatient hospital care for medically stable elderly patients?

\section{Design}

Randomised controlled trial (RCT) with 3 months follow up.

\section{Setting}

Hospital wards and their catchment areas in Bristol, UK.

\section{Patients}

241 of 383 older adults (median age 79 y, 69\% women) who were in hospital and medically stable. Inclusion criteria were availability of staff, expected positive rehabilitation, need for further hospital care if a $\mathrm{HaH}$ programme was not available, suitable home situation, agreement from the general practitioner (GP), and expected hospital stay of 1-28 days. Exclusion criterion was waiting for nursing home placement.

\section{Intervention}

After stratifying for type of admission (elective or emergency), randomisation was done to achieve twice as many patients in the $\mathrm{HaH}$ group. 160 patients allocated to the $\mathrm{HaH}$ group were discharged when they could be managed using routinely available community services. They received healthcare services between 8:30 am and 11:00 pm, or as needed. Minimal domestic services were provided. Staff included a district nurse coordinator, a registered nurse, a physiotherapist, an occupational therapist and technician, and support workers. 81 patients received usual hospital care. Follow up was $86 \%$.

\section{Main outcome and cost measures}

Mortality; physical function; quality of life; patient satisfaction; length of care; and costs for hospital and $\mathrm{HaH}$ care, for the National Health Service (NHS) and social services, and to the patient.

\begin{abstract}
Main results
The groups did not differ for mortality at 3 months $(8.3 \%$ in the HaH group $v 7.4 \%$ in the usual care group) $\{\mathrm{p}=0.8\}^{*}$, functional ability over time $(p=0.2)$, quality of life $(p=0.2)$, pain $(p=1.0)$, physical fitness $(p=0.8)$, feelings $(p=0.5)$, daily activities $(p=0.054)$, social activities $(p=0.9)$, change in health $(p=0.6)$, overall health $(p=0.9)$, or patient satisfaction $(p>0.1$ for 10 of 10 measures). In the $\mathrm{HaH}$ group, the length of care was longer (mean $16.8 \vee 12.2 \mathrm{~d}, \mathrm{p}<0.001$ ), more discussion with staff occurred $(p=0.02)$, and total costs to the NHS or social services were lower than in the hospital alone group (£2516v £3292). Costs to patients were similar in the 2 groups.
\end{abstract}

\section{Conclusions}

"Hospital at home" care was as effective and as acceptable to patients as routine hospital care for older adults. Costs were less with hospital at home care.

*Calculated from data in article.

Sources of funding: South and West National Health Service Research and Development Directorate and in part, Avon Health Authority.

For correspondence: Suzanne Richards, Research Associate, Room 2.4,Department of Social Medicine, University of Bristol, Bristol BS8 2PR, UK. Fax +44 (0)117928 7340.

\section{Commentary}

HaH care has been proposed as an effective, less costly alternative to inpatient hospital care for older adults. It provides nursing and rehabilitative services in the patient's home as a substitute for hospital care. Increasing the choices of care setting could reduce pressure on an acute care system by decreasing length of stays while avoiding unnecessary emergency hospital admissions.

Numerous studies in the US have examined the effect of home care on hospital stays. A consistent pattern of reduced hospital days across most studies suggests that $\mathrm{HaH}$ care has an important effect. ${ }^{1}$ In RCTs within the US Veterans Administration system, experimental home based, home care groups had lower costs (18$30 \%$ reductions in total costs) and utilisation ( 6 fewer hospital days) than usual care groups. ${ }^{23}$ Findings also included higher patient and caregiver satisfaction with care for the $\mathrm{HaH}$ care group. ${ }^{2-4}$ Among disabled veterans, improvements in cognitive functioning 6 months after discharge were shown. For patients with a terminal illness, no differences were found in survival, activities of daily living, cognitive functioning, or morale between groups. ${ }^{3}$

Richards et al and Shepperd et al have produced the first RCTs that explore the effectiveness and acceptability of early discharge, $\mathrm{HaH}$ programmes in the UK NHS. Both studies used similar designs, data sources, and time periods. A limitation of both studies was the use of moderate effect sizes to calculate the needed sample sizes. Previous research in the US suggests that effect size differences in outcomes among usual hospital care and
$\mathrm{HaH}$ care are small. Therefore, findings of no significant differences between the control and experimental groups for selected outcomes do not show unequivocally that one type of care could be substituted for another. A larger sample size could have addressed differences in mortality and morbidity among the groups and would have provided more convincing evidence that $\mathrm{HaH}$ care is at least as effective and can be safely substituted for hospital care.

The results of these studies are important for community based nurses providing home care for older people in terms of clinical practice, patient advocacy, and policy development. The increased costs for GP care for elderly medical patients and for patients with COPD were not (continued on page 51) 


\title{
"Hospital at home" care was generally as effective as routine hospital care for older adults
}

\author{
Shepperd S, Harwood D, Jenkinson C, et al. Randomised controlled trial comparing hospital at home care with inpatient hospital \\ care. I: three month follow up of health outcomes. BMJ 1998 Jun 13:316:1 786-91.
}

Shepperd S, Harwood D, Gray A, et al. Randomised controlled trial comparing hospital at home care with inpatient hospital care. II: cost minimisation analysis. BMJ 1998 Jun 13;316:1791-6.

\section{Question}

Is "hospital at home" $(\mathrm{HaH})$ care as effective and cost effective as inpatient hospital care for older adults?

\section{Design}

Randomised controlled trial (RCT) with 3 months follow up.

\section{Setting}

A district general hospital and its catchment area (102 general practitioners [GPs] in 26 practices) in Northampton, UK.

\section{Patients}

Adults who had hip replacements $(\mathrm{n}=86)$, knee replacements $(\mathrm{n}=86)$, hysterectomies $(\mathrm{n}=238)$, chronic obstructive airways disease (COPD) $(n=32)$, or who were elderly medical patients $(\mathrm{n}=96)$. Exclusion criteria were age $<60$ years (except for women having a hysterectomy), hysterectomy for malignancies, and an unsuitable home situation.

\section{Intervention}

After randomisation, the $\mathrm{HaH}$ group had 37 patients with hip replacement, 47 with knee replacement, 114 with hysterectomy, 15 with COPD, and 50 who were elderly medical patients. The hospital group had 49 patients with hip replacement, 39 with knee replacement, 124 with hysterectomy, 17 with COPD, and 46 who were elderly medical patients. $\mathrm{HaH}$ care included nursing, physiotherapy, occupational therapy, pathology, speech therapy, and GP care at home, with discharge decisions made by the senior nurse. Hospital patients received usual care.

\section{Main outcome and cost measures}

General health including quality of life; physical function; symptoms; hospital readmission; mortality; patient and caregiver pre- ferred form of care; and hospital, HaH, GP, patient and family, and total costs.

\section{Main results}

For hip replacement (follow up 94\%), patients who received $\mathrm{HaH}$ care had higher quality of life scores at 3 months, but no other differences, including costs. For knee replacement (follow up 93\%), more patients and caregivers in the $\mathrm{HaH}$ group preferred their care, but no other differences, including costs, occurred. For hysterectomy (follow up 83\%), more patients and fewer caregivers in the $\mathrm{HaH}$ group preferred $\mathrm{HaH}$ care, but the groups did not differ for any other outcomes except for higher costs in the HaH group (mean health services costs, £772v $£ 679, \mathrm{p}<0.01)$. Outcome assessment for patients with COPD and elderly medical patients had $<80 \%$ follow up. For patients with COPD, costs were higher with $\mathrm{HaH}$ care for median GP costs $(£ 115 v £ 15, \mathrm{p}=0.02)$ and median total health services costs $(£ 2380 v £ 1248, \mathrm{p}=0.01)$. Cost assessments for elderly medical patients showed higher median GP costs for $\mathrm{HaH}$ care $(£ 68 v £ 45, \mathrm{p}<0.01)$. Most patients preferred $\mathrm{HaH}$ care.

\section{Conclusion}

Few differences occurred in outcomes, satisfaction, and costs when "hospital at home" care was compared with usual hospital care for older adults with various diseases and conditions.

Sources of funding: RED Programme NHS Executive Anglia and Oxford; National RED Programme, Primary Secondary Care Interface; NHS Executive North Thames.

For correspondence: Sasha Shepperd, Division of Public Health and Primary Health Care, University of Oxford, Institute of Health Sciences, Headington, Oxford OX3 7LF, UK. Fax +44 (0)171 7068426. (commentary continued from page 50) surprising considering the chronic medical conditions that these patients must learn to manage. Nurses in home based care could do more to reduce the time physicians must spend with these patients by more thoroughly addressing rehabilitation needs. A rehabilitation focus helps clients and caregivers to maximise interdependent functioning and activities of daily living, promotes self care, prevents further complications and disability, and reinforces positive coping mechanisms. ${ }^{5}$ Because the $\mathrm{HaH}$ concept is relatively new in countries with a NHS, a unique opportunity may exist to develop a more rehabilitative focus for care.

The findings from the study by Shepperd $e t$ al also suggest that the needs of
$\mathrm{HaH}$ patients vary according to diagnostic category. Nurses can screen potential hospital candidates for $\mathrm{HaH}$ care. Nurses should assess readiness for discharge by considering the motivation for self care, the availability of a willing and able caregiver, and the appropriateness of the patient's home.

The findings from these studies do indicate that patients, and to some extent caregivers, prefer $\mathrm{HaH}$ care. If it can be shown that no adverse consequences follow $\mathrm{HaH}$ care and that the care is at least as effective as usual hospital care, the preferences of patients for their care location should be respected. Nurses are in a strong position to advocate for patient preferences within the healthcare system. By advocating for patient and family pref- erences, and insisting that these preferences be included in further research, nurses will make a substantial contribution to policy and funding decisions.

Deborah Messecar, RN, PhD Assistant Professor Population Based Care Department School of Nursing Oregon Health Sciences University Portland, Oregon, USA

1 Hughes SL, Ulasevich A, Weaver, FM, et al. Health Serv Res 1997;32:415-32.

2 Cummings JE, Hughes SL, Weaver FM, et al. Arch Intern Med 1990;150:1274-80.

Hughes SL, Cummings JE, Weaver FM, et al. Health Serv Res 1992;26:801-17.

4 Hughes SL, Cummings JE, Weaver FM, et al. Med Care 1990;28:135-45.

5 Neal LJ, editor. Rehabilitation nursing in the home health setting. Chicago, Illinois: Association of Rehabilitation Nurses, 1998. 